\title{
Molecular diversity analysis in hexaploid wheat (Triticum aestivum L.) and two Aegilops species (Aegilops crassa and Aegilops cylindrica) using CBDP and SCOT markers
}

\author{
Ghazal Ghobadi, Alireza Etminan * (D), Ali Mehras Mehrabi and Lia Shooshtari
}

\begin{abstract}
Background: Evaluation of genetic diversity and relationships among crop wild relatives is an important task in crop improvement. The main objective of the current study was to estimate molecular variability within the set of 91 samples from Triticum aestivum, Aegilops cylindrica, and Aegilops crassa species using 30 CAAT box-derived polymorphism (CBDP) and start codon targeted (SCOT) markers.

Results: Fifteen SCOT and Fifteen CBDP primers produced 262 and 298 fragments which all of them were polymorphic, respectively. The number of polymorphic bands (NPB), polymorphic information content (PIC), resolving power (Rp), and marker index (MI) for SCoT primers ranged from 14 to $23,0.31$ to $0.39,2.55$ to 7.49 , and 7.56 to 14.46 with an average of $17.47,0.34,10.44$, and 5.69, respectively, whereas these values for CBDP primers were 15 to $26,0.28$ to $0.36,3.82$ to 6.94 , and 4.74 to 7.96 with a mean of $19.87,0.31,5.35$, and 6.24 , respectively. Based on both marker systems, analysis of molecular variance (AMOVA) indicated that the portion of genetic diversity within species was more than among them. In both analyses, the highest values of the number of observed $(\mathrm{Na})$ and effective alleles ( $\mathrm{Ne})$, Nei's gene diversity ( $\mathrm{He})$, and Shannon's information index (I) were estimated for Ae. cylindrica species.

Conclusion: The results of cluster analysis and population structure showed that SCOT and CBDP markers grouped all samples based on their genomic constitutions. In conclusion, the used markers are very effective techniques for the evaluation of the genetic diversity in wild relatives of wheat.
\end{abstract}

Keywords: Genetic diversity, Gene-based markers, Population structure, Polymorphism information content

\section{Background}

Based on the International Grains Council's report (2019) [1], the world needs more one billion tons of wheat for the next 4 years $(\sim 2024)$. It seems that this demand is fulfilled through conventional breeding programs [2]. However, there is a main concern among breeders that the genetic background of cultivated genotypes is narrowed

\footnotetext{
* Correspondence: alietminan55@yahoo.com

Department of plant breeding and Biotechnology, Kermanshah Branch, Islamic Azad University, Kermanshah, Iran
}

by consecutive breeding cycles and remaining variability in its gene pool is inadequate for future breeding programs [3]. Therefore, the expansion of the genetic base of this important cereal is necessary. The genus Aegilops as the most important wheat gene pool can contribute to obtaining favorable traits in breeding programs. This genus includes 22 species at the three di-, tetra-, and hexaploid levels as well as with various genetic structures such as the U, M, S, B, D, N, X, and T genomes [4]. Numerous reports have revealed that different Aegilops species can be introducing desirable agronomic properties and 
breeding potential which induce resistance to various biotic and abiotic stresses [5-19]. One of the first fundamental step in each breeding program is the estimate of genetic diversity. Indeed, accurate investigation of the level of genetic diversity can be important in its breeding programs for characterizing ideal parental plants to provide segregating progenies for further basic analysis and selection [20].

Investigation of genetic diversity in wheat and its wild relatives has been done through agro-morphological characters, properties, and molecular markers techniques. Despite the botanical characters and agro-morphological traits being usually used to dissect genetic diversity, they are not completely successful due to environmental influences. In contrast, molecular markers as the genetic tools provide important information regarding the genetic structure and phylogenetic relationships among different plant species. These molecular tools expose genetic differences or similarities in better information without interference from environmental factors [21].

Various molecular marker techniques such as AFLP (amplified fragment length polymorphism), RAPD (randomly amplified polymorphic DNA), SSR (simple sequence repeat), ISSR (inter simple sequence repeats), DArT (diversity arrays technology), etc. are currently available for the evaluation of genetic population analysis, association analysis, and QTL mapping studies. CAAT box-derived polymorphisms (CBDP) and start codon targeted (SCoT) polymorphisms are two new reproducible markers that are based on the short conserved region in plant genes [22, 23]. These techniques have been successfully used in genetic analyses in different plant species [24-32]. The present research is focused on the estimation of genetic diversity and population structure in a set of Iranian bread wheat genotypes and two Aegilops species using SCoT and CBDP markers.

\section{Methods}

\section{Plant materials and DNA extraction}

The plant materials consisted of 91 accessions belonging to Ae. cylindrica, Ae. crassa, and T. aestivum L. species. The genetic resources of wild species were collected from the natural habitats in Iran. and the seeds of all accessions were deposited in the Ilam University Gene bank with specific voucher numbers. The genetic composition and gene bank codes are presented in Table 1 . Total genomic DNA of investigated accessions was isolated from fresh leaves based on CTAB protocol [33]. The quality of extracted DNA was tested by $0.8 \%$ agarose gel electrophoresis.

Polymerase chain reaction using SCOT and CBDP markers A total of 15 SCoT primers(Table 2) were selected based on [21]. Polymerase chain reactions (PCRs) were conducted in a volume of $20 \mu \mathrm{L}$ and consisted of $2 \mu \mathrm{L}$ of DNA, $2 \mu \mathrm{L}$ of each primer, $10 \mu \mathrm{L}$ master mix PCR (ready-to-use PCR master mix $2 \times$ ), and $6 \mu \mathrm{L} \mathrm{ddH}_{2} \mathrm{O}$. The amplification conditions included an initial denaturation step of $5 \mathrm{~min}$ at $94{ }^{\circ} \mathrm{C}$, followed by 45 cycles of 45 $\mathrm{s}$ at $94{ }^{\circ} \mathrm{C}, 1 \mathrm{~min}$ at $45{ }^{\circ} \mathrm{C}$, and $3 \mathrm{~min}$ at $72{ }^{\circ} \mathrm{C}$ with a final extension at $72{ }^{\circ} \mathrm{C}$ for $7 \mathrm{~min}$. Produced fragments were separated by gel electrophoresis in $1.5 \%$ agarose. In CBDP analysis, 15 primers were designed based on [23], (Table 2). Similar to SCoT assay, each PCR reaction was amplified in a volume of $20 \mu \mathrm{L}$ and contained $2 \mu \mathrm{L}$ DNA, $2 \mu \mathrm{L}$ of each primer, $6 \mu \mathrm{l}$ double-distilled water, and $10 \mu \mathrm{l}$ master mix. All reactions were carried out as follows: an initial denaturation step at $94{ }^{\circ} \mathrm{C}$ for $5 \mathrm{~min}$, followed by 45 cycles of denaturation at $94{ }^{\circ} \mathrm{C}$ for $45 \mathrm{~s}$, primer annealing at $56{ }^{\circ} \mathrm{C}$ for $45 \mathrm{~s}$, and primer elongation at $72{ }^{\circ} \mathrm{C}$ for $90 \mathrm{~s}$; the final extension at $72{ }^{\circ} \mathrm{C}$ was held for $10 \mathrm{~min}$. All products were run on a $1.5 \%$ agarose gel. In both systems, all amplified fragments were stained with Safestaine-II and finally photographed using a gel documentation device.

\section{Data analysis}

All the observed bands in SCoT and CBDP profiles were scored as 1 and 0 on the basis of the attendance presence and absence of the amplified fragments, respectively. To determination of efficiency the selected primers, five informativeness indices, such as the number of polymorphic bands (NPB), polymorphism information content (PIC), resolving power $(\mathrm{Rp})$, and marker index $(\mathrm{MI})$, were estimated. Partitioning the genetic diversity among and within species was done through analysis of molecular variance (AMOVA). Genetic variation parameters including the number of observed $(\mathrm{Na})$ and effective alleles $(\mathrm{Ne})$, Shannon's information index (I), Nei's gene diversity $(\mathrm{He})$, and percentage of polymorphic loci $(P P L)$ were calculated for comparing the level of genetic diversity among different species. All genetic parameters were calculated using GenAlEx software [34]. Cluster analysis was computed based on the Jaccard's dissimilarity matrix to the grouping of the investigated Aegilops accessions using DARwin software ver. 6.0.13 [35]. Population structure analysis was carried out using STRUCTURE software [36]. To obtain the optimum number of subpopulations, seven independent runs were determined, so in each run, the values of burn-in period and MCMC factors were 50,000. Then, the results of structure analysis were subjected to an estimate of subpopulations $(\Delta K)$ using the STRUCTURE HARVES TER software [37].

\section{Results}

SCoT and CBDP polymorphism

All tested SCoT and CBDP primers were polymorphic and reproducible. The summary of estimated 
Table 1 List of the 91 investigated Triticum and Aegilops accessions

\begin{tabular}{|c|c|c|c|c|c|c|c|c|}
\hline No. & Genbank code & Species & No. & Genbank code & Species & No. & Genbank code & Species \\
\hline 1 & IUGB-00615 & T. aestivum & 32 & NPGBI-365 & Ae. crassa & 62 & IUGB-00078 & Ae. cylindrica \\
\hline 2 & IUGB-00597 & T. aestivum & 33 & NPGBI-310 & Ae. crassa & 63 & IUGB-00090 & Ae. cylindrica \\
\hline 3 & IUGB-00604 & T. aestivum & 34 & NPGBI-309 & Ae. crassa & 64 & IUGB-00406 & Ae. cylindrica \\
\hline 4 & IUGB-00603 & T. aestivum & 35 & NPGBI-2066 & Ae. crassa & 65 & IUGB-00258 & Ae. cylindrica \\
\hline 5 & IUGB-00576 & T. aestivum & 36 & NPGBI-1589 & Ae. crassa & 66 & IUGB-00248 & Ae. cylindrica \\
\hline 6 & IUGB-00618 & T. aestivum & 37 & NPGBI-792 & Ae. crassa & 67 & IUGB-00388 & Ae. cylindrica \\
\hline 7 & IUGB-01845 & T. aestivum & 38 & NPGBI-947 & Ae. crassa & 68 & IUGB-01592 & Ae. cylindrica \\
\hline 8 & IUGB-00518 & T. aestivum & 39 & NPGBI-1485 & Ae. crassa & 69 & IUGB-00202 & Ae. cylindrica \\
\hline 9 & IUGB-00593 & T. aestivum & 40 & NPGBI-1508 & Ae. crassa & 70 & IUGB-00201 & Ae. cylindrica \\
\hline 10 & IUGB-00570 & T. aestivum & 41 & NPGBI-384 & Ae. crassa & 71 & IUGB-00406 & Ae. cylindrica \\
\hline 11 & IUGB-00575 & T. aestivum & 42 & NPGBI-2112 & Ae. crassa & 72 & IUGB-00229 & Ae. cylindrica \\
\hline 12 & IUGB-01846 & T. aestivum & 43 & NPGBI-720 & Ae. crassa & 73 & IUGB-00090 & Ae. cylindrica \\
\hline 13 & IUGBI-00577 & T. aestivum & 44 & NPGBI-2063 & Ae. crassa & 74 & IUGB-00270 & Ae. cylindrica \\
\hline 14 & IUGBI-00589 & T. aestivum & 45 & NPGBI-911 & Ae. crassa & 75 & IUGB-00059 & Ae. cylindrica \\
\hline 15 & IUGB-00573 & T. aestivum & 46 & NPGBI-1699 & Ae. crassa & 76 & IUGB-00132 & Ae. cylindrica \\
\hline 16 & IUGB-00600 & T. aestivum & 47 & NPGBI-587 & Ae. crassa & 77 & IUGB-00095 & Ae. cylindrica \\
\hline 17 & IUGB-00578 & T. aestivum & 48 & NPGBI-794 & Ae. crassa & 78 & IUGB-00062 & Ae. cylindrica \\
\hline 18 & IUGB-00602 & T. aestivum & 49 & NPGBI-944 & Ae. crassa & 79 & IUGB-01359 & Ae. cylindrica \\
\hline 19 & IUGB-00586 & T. aestivum & 50 & NPGBI-2117 & Ae. crassa & 80 & IUGB-01238 & Ae. cylindrica \\
\hline 20 & IUGB-00598 & T. aestivum & 51 & NPGBI-1742 & Ae. crassa & 81 & IUGB-00239 & Ae. cylindrica \\
\hline 21 & IUGB-00515 & T. aestivum & 52 & NPGBI-598 & Ae. crassa & 82 & IUGB-00078 & Ae. cylindrica \\
\hline 22 & IUGB-01847 & T. aestivum & 53 & NPGBI-744 & Ae. crassa & 83 & IUGB-00065 & Ae. cylindrica \\
\hline 23 & IUGB-00534 & T. aestivum & 54 & NPGBI-1473 & Ae. crassa & 84 & IUGB-00391 & Ae. cylindrica \\
\hline 24 & IUGB-00613 & T. aestivum & 55 & NPGBI-1522 & Ae. crassa & 85 & IUGB-00241 & Ae. cylindrica \\
\hline 25 & IUGB-00590 & T. aestivum & 56 & NPGBI-675 & Ae. crassa & 86 & IUGB-00153 & Ae. cylindrica \\
\hline 26 & IUGB-00606 & T. aestivum & 57 & NPGBI-730 & Ae. crassa & 87 & IUGB-00390 & Ae. cylindrica \\
\hline 27 & IUGB-00599 & T. aestivum & 58 & NPGBI-689 & Ae. crassa & 88 & IUGB-00399 & Ae. cylindrica \\
\hline 28 & IUGB-01840 & T. aestivum & 59 & NPGBI-50067 & Ae. crassa & 89 & IUGB-00201-S1 & Ae. cylindrica \\
\hline 29 & IUGB-00532 & T. aestivum & 60 & NPGBI-50174 & Ae. crassa & 90 & IUGB-01592 & Ae. cylindrica \\
\hline 30 & IUGB-00580 & T. aestivum & 61 & IUGB-00062 & Ae. cylindrica & 91 & IUGB-00388-S1 & Ae. cylindrica \\
\hline 31 & NPGBI-976 & Ae. crassa & & & & & & \\
\hline
\end{tabular}

IUGB Ilam University Genebank, NPGBI The national Plant Genebank of Iran

informativeness indices for each primer is presented in Table 2. The 15 used SCoT primers amplified 262 distinct fragments, which all of them were polymorphic. The number of bands per primer varied between 14 (SCoT-12, SCoT-13, and SCoT-15) and 23 (SCoT-20 and SCoT-21) with a mean of 17.47 per primer. The average Rp index was 10.44, and primers SCoT-15 and SCoT-5 showed the highest (14.46) and lowest (7.56) values, respectively. The MI index ranged from 2.55 to 7.49 with a mean of 5.69 per primer and the SCoT-19 primer indicated the highest value, while SCoT-13 showed the lowest MI value. PIC index varied between 0.31 and 0.39 with a mean of 0.34 . The primer SCoT-1 with the highest value was recognized from others as the informativeness primer, whereas primers SCoT-6, SCoT-20, and SCoT-21 showed the lowest values.

In the CBDP assay, 15 polymorphic primers amplified 298 fragments. Primers CBDP-12 and CBDP-10 amplified the maximum (26) and minimum (15) numbers of polymorphic fragments. Rp index ranged from 3.82 and 6.94 with an average of 5.35 per primer. CBDP-8 and CBDP-11 showed the highest and lowest values for this index than other primers. The MI index varied between 4.74 and 7.96 with an average of 6.24 per primer, and the highest and lowest values were estimated for CBDP7 and CBDP-10 primers, respectively. The mean of PIC 
Table 2 List of used SCOT and CBDP primers and their calculated informativeness parameters on 91 investigated Triticum and Aegilops

\begin{tabular}{|c|c|c|c|c|c|c|}
\hline Primer & Sequence & TB & PB & PIC & Rp & MI \\
\hline SCOT-2 & CAACAATGGCTACCACCC & 18 & 18 & 0.39 & 12.04 & 7.09 \\
\hline SCoT-3 & CAACAATGGCTACCACCG & 16 & 16 & 0.33 & 8.84 & 5.29 \\
\hline CoT-5 & CAACAATGGCTACCACGA & 16 & 16 & 0.26 & 7.56 & 4.17 \\
\hline SCOT-6 & CAACAATGGCTACCACGC & 19 & 19 & 0.31 & 9.69 & 5.95 \\
\hline CoT-7 & CAACAATGGCTACCACGG & 19 & 19 & 0.34 & 9.03 & 6.42 \\
\hline SCOT-12 & ACGACATGGCGACCAACG & 14 & 14 & 0.37 & 10.13 & 5.21 \\
\hline CoT-13 & ACGACATGGCGACCATCG & 14 & 14 & 0.32 & 7.93 & 2.55 \\
\hline SCOT-14 & ACGACATGGCGACCACGC & 16 & 16 & 0.37 & 10.00 & 3.70 \\
\hline SCoT-15 & ACGACATGGCGACCGCGA & 14 & 14 & 0.37 & 14.46 & 5.22 \\
\hline CoT-16 & CCATGGCTACCACCGGCC & 16 & 16 & 0.37 & 9.76 & 5.90 \\
\hline SCoT-17 & CATGGCTACCACCGGCCC & 16 & 16 & 0.35 & 11.78 & 5.63 \\
\hline SCoT-18 & ACCATGGCTACCACCGCG & 17 & 17 & 0.38 & 10.84 & 6.43 \\
\hline SCoT-19 & GCAACAATGGCTACCACC & 21 & 21 & 0.36 & 12.59 & 7.49 \\
\hline CoT-20 & AACCATGGCTACCACCGC & 23 & 23 & 0.31 & 10.79 & 7.06 \\
\hline COT-21 & CACCATGGCTACCACCAT & 23 & 23 & 0.31 & 11.12 & 7.19 \\
\hline Mean & & 17.47 & 17.47 & 0.34 & 10.44 & 5.69 \\
\hline BDP-1 & TGAGCACGATCCAAT AGC & 19 & 19 & 0.29 & 4.69 & 5.47 \\
\hline CBDP-2 & TGAGCACGATCCAATAAT & 20 & 20 & 0.28 & 5.65 & 5.65 \\
\hline CBDP-3 & TGAGCACGATCCAAT ACC & 21 & 21 & 0.31 & 5.45 & 6.51 \\
\hline CBDP-4 & TGAGCACGATCCAAT AAG & 15 & 15 & 0.36 & 6.27 & 5.33 \\
\hline CBDP-5 & TGAGCACGATCCAAT CTA & 16 & 16 & 0.33 & 4.12 & 5.24 \\
\hline CBDP-6 & TGAGCACGATCCAAT CAG & 15 & 15 & 0.36 & 4.74 & 5.33 \\
\hline 7 & TGAGCACGATCCAAT CGA & 22 & 22 & 0.36 & 6.52 & 7.96 \\
\hline CBDP-8 & TGAGCACGATCCAAT CGG & 25 & 25 & 0.33 & 6.95 & 8.15 \\
\hline CBDP-9 & TGAGCACGATCCAAT GAT & 23 & 23 & 0.29 & 5.09 & 6.78 \\
\hline CBDP-10 & TGAGCACGATCCAAT GTT & 15 & 15 & 0.32 & 4.69 & 4.75 \\
\hline CBDP-11 & TGAGCACGATCCAAT TGC & 18 & 18 & 0.28 & 3.82 & 5.07 \\
\hline CBDP-12 & TGAGCACGATCCAATATA & 26 & 26 & 0.31 & 7.00 & 8.07 \\
\hline CBDP-13 & TGAGCACGATCCAATGAG & 20 & 20 & 0.29 & 5.08 & 5.84 \\
\hline CBDP-14 & TGAGCACGATCCAATGCG & 22 & 22 & 0.33 & 5.59 & 7.36 \\
\hline CBDP-15 & TGAGCACGATCCAATTGA & 21 & 21 & 0.29 & 4.52 & 6.05 \\
\hline Mean & & 19.87 & 19.87 & 0.31 & 5.35 & 6.24 \\
\hline
\end{tabular}

$\mathrm{TB}, \mathrm{PB}, \mathrm{PIC}, \mathrm{Rp}$, and MI indicate total amplified bands, polymorphic amplified bands, polymorphism information content, resolving power and marker index parameters, respectively

index was 0.31 and it ranged from 0.28 (CBDP-2 and CBDP-11) to 0.36 (CBDP-4, CBDP-6, and CBDP-7) (Table 2).

\section{Genetic diversity in Aegilops species}

The results of the AMOVA analysis are shown in Fig. 1. Based on both marker systems, a significant difference within species was observed. Based on SCoT data, the portion of genetic variance within and between species were 78 and $22 \%$, respectively, while based on CBDP data these portions were 80 and $20 \%$, respectively. Moreover, based on both marker systems, there was a high level of genetic differentiation $\left(G_{S T}\right)$ among the studied populations (Table 3). Besides, the values of gene flow $\mathrm{Nm}$ ) parameter for SCoT and CBDP markers were less than 1, showing a genetic isolation among different species. A summary of the estimated genetic variation parameters based on SCoT and CBDP markers is presented in Table 3. In SCoT analysis, mean values of $\mathrm{Na}, \mathrm{Ne}, \mathrm{I}, \mathrm{He}$, and PPL were $1.73,1.35,0.35,0.22$, and 86.01 , respectively. The highest values for $\mathrm{Na}$ and $P P L$ were observed for $T$. aestivum species, while the highest values for $\mathrm{Na}, \mathrm{Ne}$, I, and $\mathrm{He}$ were recorded for Ae. cylindrica species. On the other hand, the results based on CBDP data indicated that the average values of all genetic parameters were lower than SCoT data $(\mathrm{Na}=1.68$, $\mathrm{Ne}=1.33, I=0.33, \mathrm{He}=0.21$, and $P P L=83.78 \%)$. The highest values of all parameters were recorded for $A e$. cylindrica species.

\section{Grouping of Aegilops accessions and population structure analysis}

The dendrogram rendered using the neighbor-joining algorithm (NJ) based on the SCoT data sets clustered all investigated samples into three main groups. The first, second, and third clusters consisted of 58, 29, and 4 samples, respectively (pairwise genetic distance coefficients are not shown). The first cluster (GI) was further divided into two sub-clusters (sub-I and sub-II). Sub-I included 21 accessions from Ae. crassa and 9 accessions from Ae. cylindrica, while sub-II consisted of 9 and 22 accessions from Ae. cylindrica and Ae. crassa, respectively. All $T$. aestivum accessions (except no. 1) were placed in the second cluster (GII). Three samples from Ae. cylindrica (nos. 89, 90, and 91) along with one sample of T. aestivum (no. 1) created the third cluster (GIII) (Fig. 2a). The dendrogram obtained using the CBDP data set indicated that all Aegilops samples were grouped into three main clusters. The first cluster (GI) embraced all bread wheat accessions. The second cluster (GII) consisted of 22 samples from Ae. cylindrica along with 21 samples from Ae. crassa species. The remaining samples from Ae. cylindrica and Ae. crassa were grouped in the third cluster (GIII) (Fig. 2b).

In the population structure analysis, the maximum $\Delta \mathrm{K}$ for both data sets were observed at $K=3$, with accessions falling into three subpopulations (Fig. 3a). In both analyses, the threshold level of membership for each sample in subpopulations was determined $\geq 0.5$. Based on SCoT data, 20 samples of T. aestivum created the first subpopulation. All accessions of Ae. crassa except nos. 59 and 60 along with nine samples belonging to $T$. aestivum were clustered into the second subpopulation. 

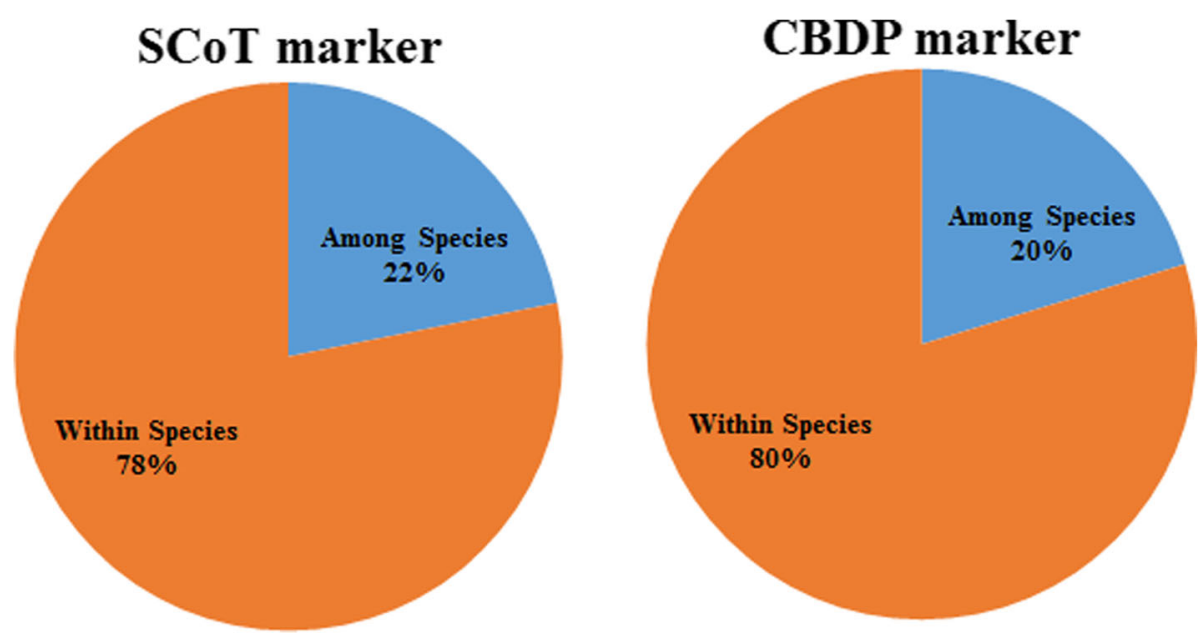

Fig. 1 Results of analysis of molecular variance using SCOT and CBDP markers in the 91 investigated wheat's landraces and wild relatives

Two samples from Ae. crassa (nos. 59 and 60) and all Ae. cylindrica were the third subpopulation. One sample (accession no. 2 from T. aestivum) was categorized into an admixed group (Fig. 3a). In CBDP analysis, the optimum number of subpopulations was revealed to be $K=3$, which indicated that all of the samples can be grouped into three main subpopulations with an admixed group. Out of 30 samples from $T$. aestivum species, 22 samples were placed in subpopulation I, five samples (Nos. 20, 22, 28, 29, and 30) fell into subpopulation II, and three samples (nos. 21, 24, and 25) along with one sample from Ae. crassa species (no. 59) were placed in the admixed group, respectively. All samples from Ae. crassa were separated from other samples and created subpopulation II. However, one sample from Ae. cylindrica was categorized in this subpopulation. Finally, the remaining Ae. cylindrica (30 samples) were assigned to subpopulation III (Fig. 3b). The results obtained by cluster analysis and population structure are generally supported by the principal coordinate analysis (PCoA). As shown in Fig. 4, all investigated samples were grouped into two main clusters using SCoT and CBDP markers. In both biplots, all accessions belonging to $T$. aestivum species were placed into the same cluster; however, all Ae. cylindrica and Ae. crassa fell into the same cluster together.

\section{Discussion}

Increasing the crop adaptability to climate change and ensuring food security for the next century are two critical scenarios which reveal the importance of genetic diversity in crop wild relatives. Among cereal crops, wheat has a rich gene pool, including many wild relatives with various genomic constructions. This feature has enabled wheat breeders to use them as a main source of important agronomic characters and ideal genes which are involved in tolerance to different biotic and abiotic stresses [38]. Therefore, investigation of molecular variability in wild relatives of wheat is a key task in exploring novel genes or even alleles for future breeding programs [39]. Molecular analysis study using DNA-based markers is an efficient approach to estimate genome diversity and population structure that has been used repeatedly in many plants. In the current study, CBDP and SCoT marker techniques served to investigate genetic diversity between and within two Aegilops species along with local bread wheat genotypes. All amplified fragments using both marker systems were polymorphic, which

Table 3 Estimated genetic parameters in bread wheat and two Aegilops using SCOT and CBDP markers

\begin{tabular}{|c|c|c|c|c|c|c|c|c|c|}
\hline Marker & Species & Sample size & $\mathrm{Na}$ & $\mathrm{Ne}$ & $\mathrm{I}$ & $\mathrm{He}$ & PPL & $G_{S T}$ & $\mathrm{Nm}$ \\
\hline \multirow[t]{3}{*}{ SCOT } & T. aestivum & 30 & 1.79 & 1.35 & 0.36 & 0.22 & $89.31 \%$ & 0.46 & 0.78 \\
\hline & Ae. crassa & 30 & 1.71 & 1.33 & 0.33 & 0.21 & $84.35 \%$ & & \\
\hline & Ae. cylindrica & 31 & 1.71 & 1.39 & 0.37 & 0.24 & $84.35 \%$ & & \\
\hline \multirow[t]{3}{*}{ CBDP } & T. aestivum & 30 & 1.67 & 1.36 & 0.35 & 0.22 & $83.56 \%$ & 0.51 & 0.82 \\
\hline & Ae. crassa & 30 & 1.60 & 1.29 & 0.29 & 0.18 & $78.86 \%$ & & \\
\hline & Ae. cylindrica & 31 & 1.79 & 1.36 & 0.36 & 0.23 & $88.93 \%$ & & \\
\hline
\end{tabular}

$\mathrm{Na}, \mathrm{Ne}, \mathrm{I}, \mathrm{He}, \mathrm{PPL}, \mathrm{G}_{\mathrm{ST}}$, and Nm indicate the number of observed alleles, number of effective alleles, Shannon's information index, Nei's genetic diversity, percentage polymorphism loci, the coefficient of genetic differentiation, and gene flow respectively 


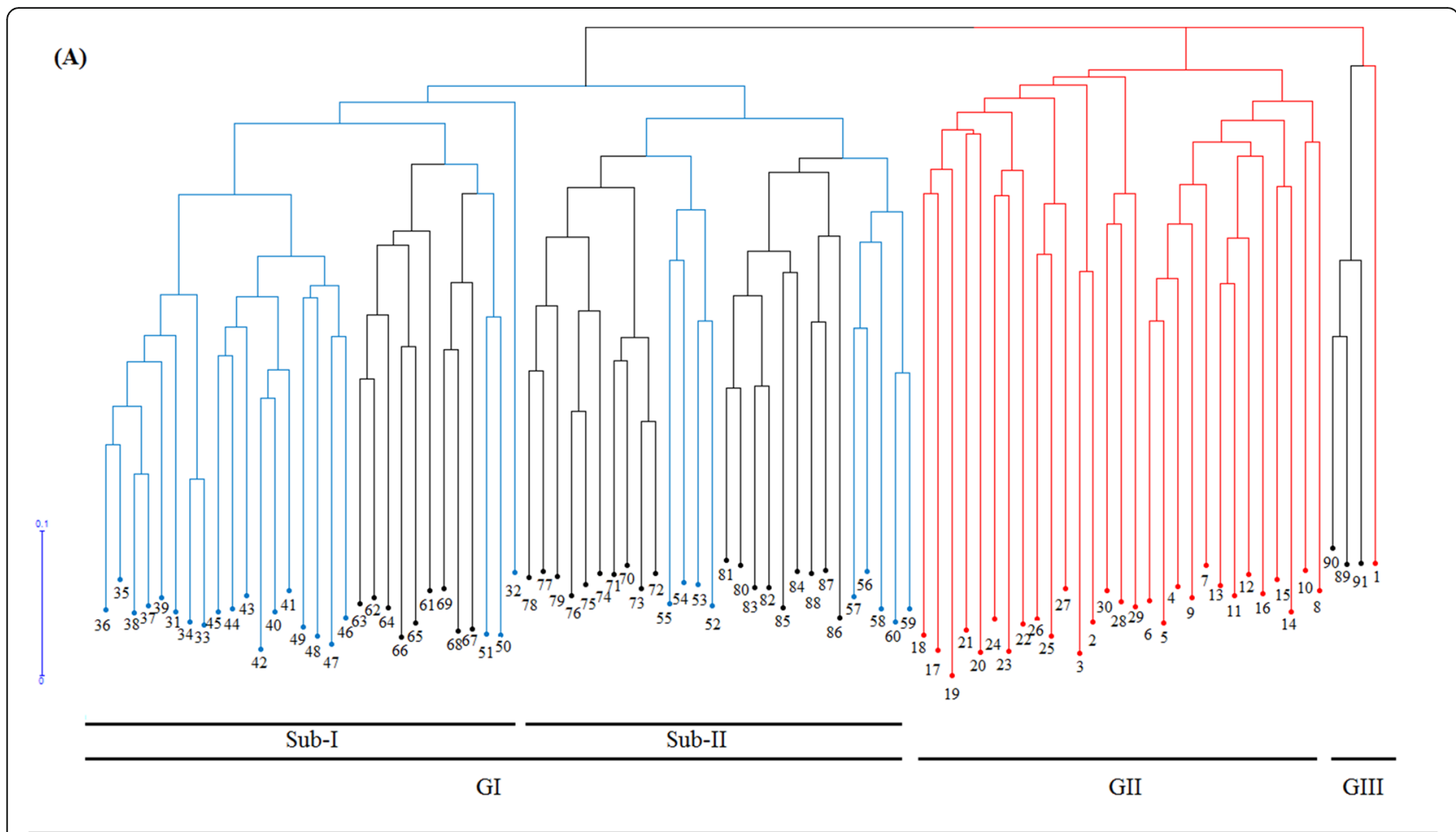

(B)

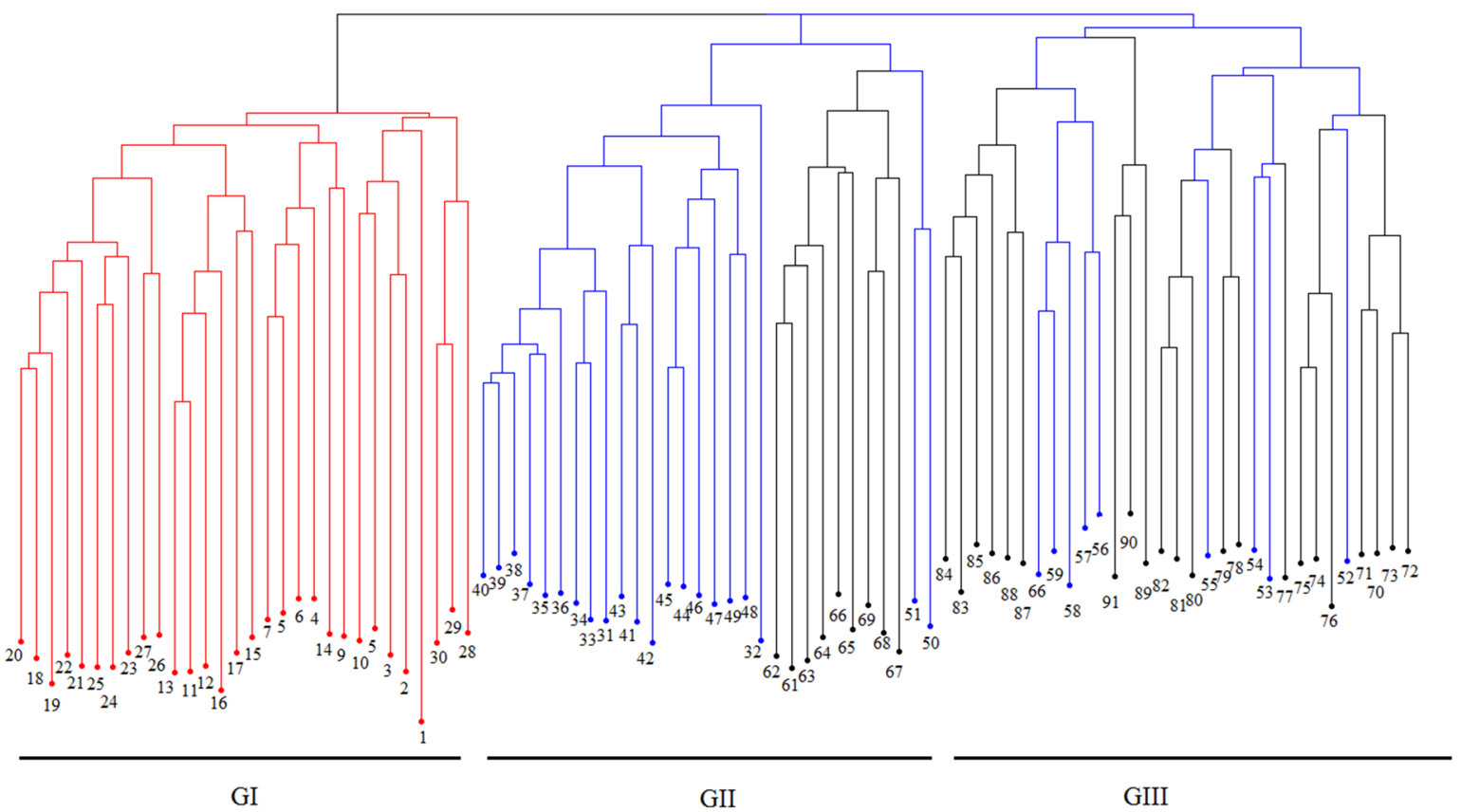

Fig. 2 Hierarchical clustering patterns of investigated accessions based on Jaccard's dissimilarity coefficient using SCoT (panel a) and CBDP (panel b) markers. Red, black and blue lines represent the T. aestivum, Ae. cylindrica and Ae. crassa accessions, respectively

confirmed that the used markers are a powerful tool for further genetic diversity analyses and classify the investigated samples (Table 2). Pour-Aboughadareh et al. [40] used SCoT markers to analyze polymorphism of four Triticum species including T. aestivum, T. durum, T. urartu, and T. boeoticum and obtained $97.59 \%$ of polymorphism fragments. Analogous works on wild relatives of bread wheat and different Aegilops species were conducted by Pour-Aboughadareh et al. [29] and Etminan et al. [41] and these researchers reported the high level 

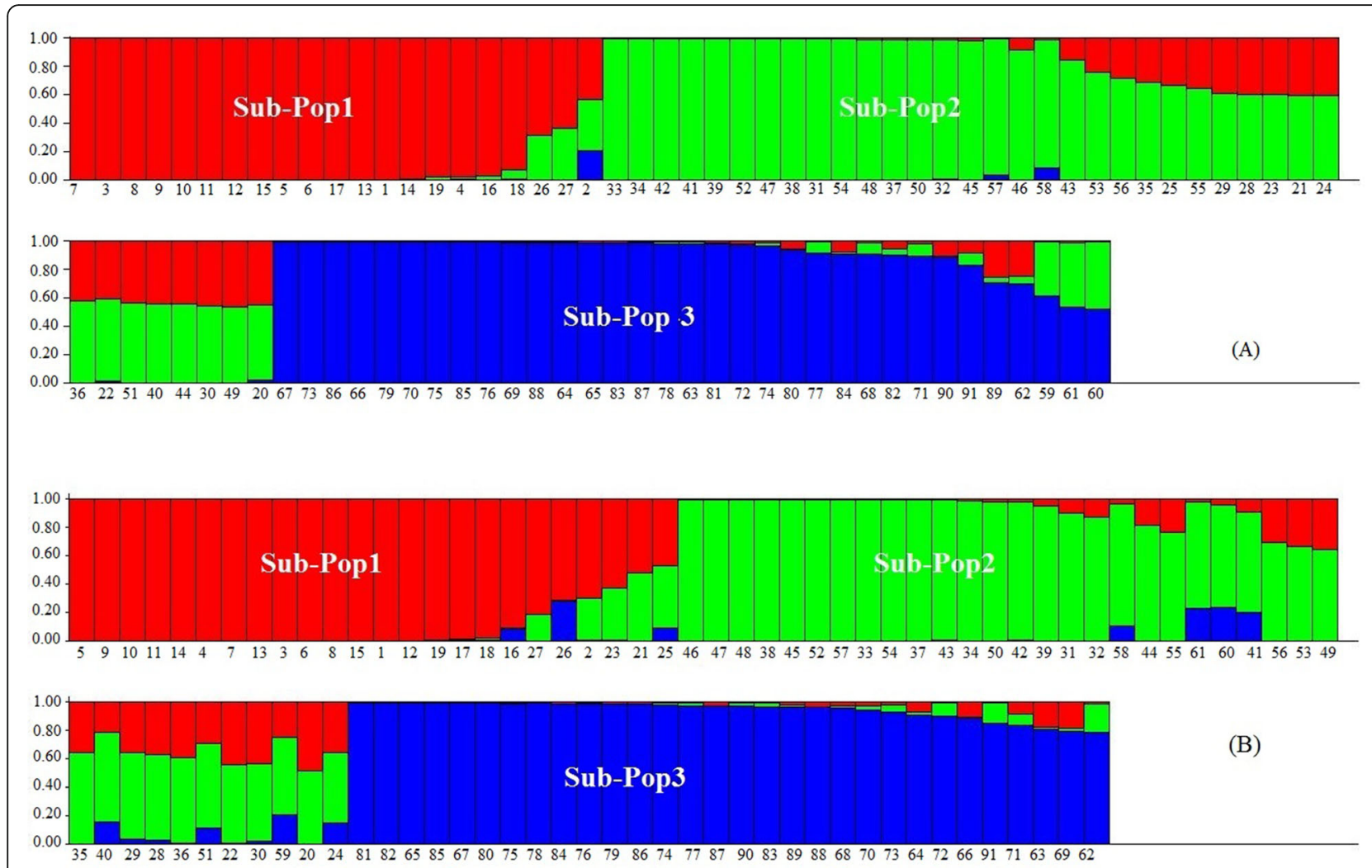

Fig. 3 Population structure of 91 Triticum and Aegilops accessions using SCOT (panel a) and CBDP (panel b)

of polymorphism using SCoT and CBDP markers. In addition to percentage polymorphism, resolving power (Rp) and polymorphism information content (PIC) are the important indices of marker usefulness used for comparison efficiency of markers for genetic analyses [29]. PIC depends on the number of detectable alleles and is described as the probability of a primer in identifying polymorphism between samples. On the other hand, $\mathrm{Rp}$ shows the discriminatory ability of the used primers to produce informative fragments [42]. Average amounts of PIC and RP confirmed the usefulness of the selected primers for analysis of genetic diversity and grouping the samples belonging to the different species of the Triticum and Aegilops genera (Table 2). Likewise, Etminan et al. [43] investigated accessions of the $T$. durum and obtained a PIC of 0.31 and Rp of 9.16 using CBDP markers. Also, in another study, these authors used fifteen CBDP primers for dissection of molecular variability in different Aegilops and Triticum species and reported a high level of polymorphism and discriminatory of the used markers (PIC $=0.47$ and $\mathrm{Rp}=11.19$ ). Nowak et al. [2] analyzed three Aegilops species (Ae. crassa, Ae. neglecta, and Ae. juvenalis) using REMP and ISSR markers. The authors indicated that the used markers were efficient systems for evaluating the genetic diversity and also reported that the Aegilops species have a high level of genome variability which can serve as an ideal gene pool for discovering useful genes.

The result of AMOVA revealed that genetic diversity observed within species ( $\mathrm{SCoT}=78 \%$ and $\mathrm{CBDP}=80 \%$ ) is more to that found among them (SCoT $=22 \%$ and CBDP $=80 \%)$, suggesting all accessions in each species have a wide genetic differentiation (Fig. 1). This finding is in accordance with those of the previous reports that showed the high level of diversity in Aegilops species through different DNA markers [2, 29, 41, 44-48]. Our results indicate the accessions from three different species are genetically different from each other. As shown in Table 3, the highest values of the genetic variation indices (especially $\mathrm{Na}, \mathrm{Ne}, \mathrm{I}$, and $\mathrm{He}$ ) were observed for Ae. cylindrica species using both marker systems. The higher level of diversity in this species might be referred to as the frequency of allelic variation of this species being affected by different climatic conditions [2]. Several studies considered Ae. cylindrica as novel sources of tolerance to abiotic stresses for further wheat breeding programs [49]. Pour-Aboughadareh et al. [29] reported that Ae. cylindrica has the highest level of genetic diversity 


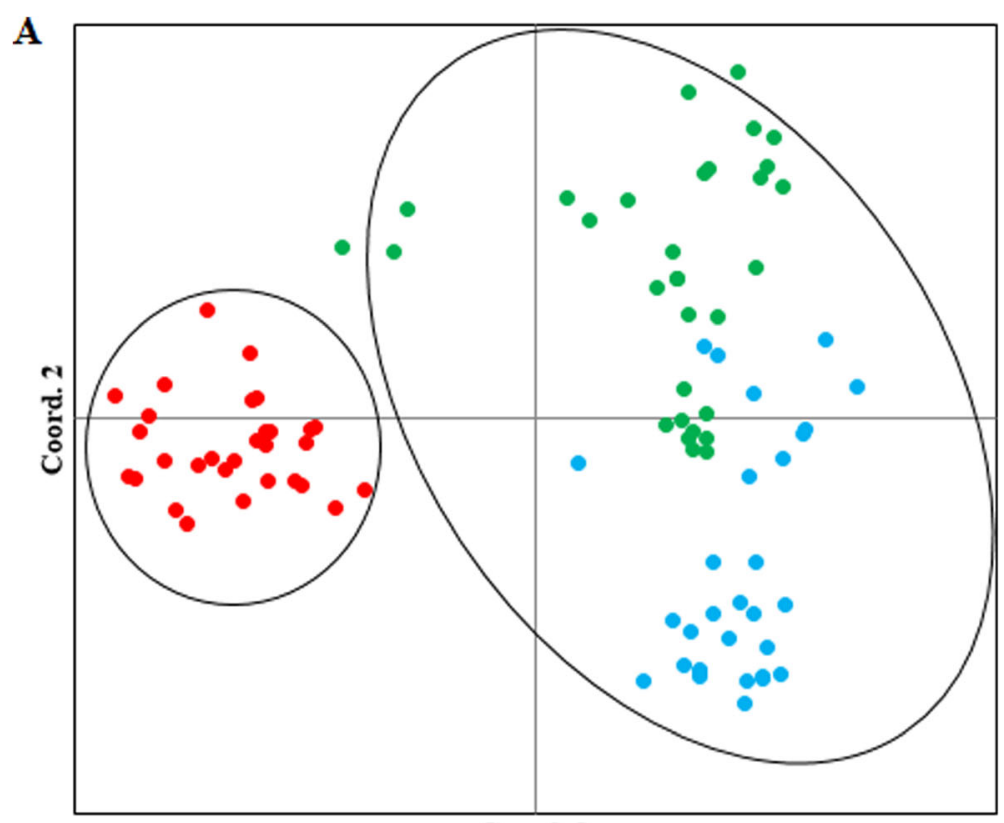

B

Coord. 1

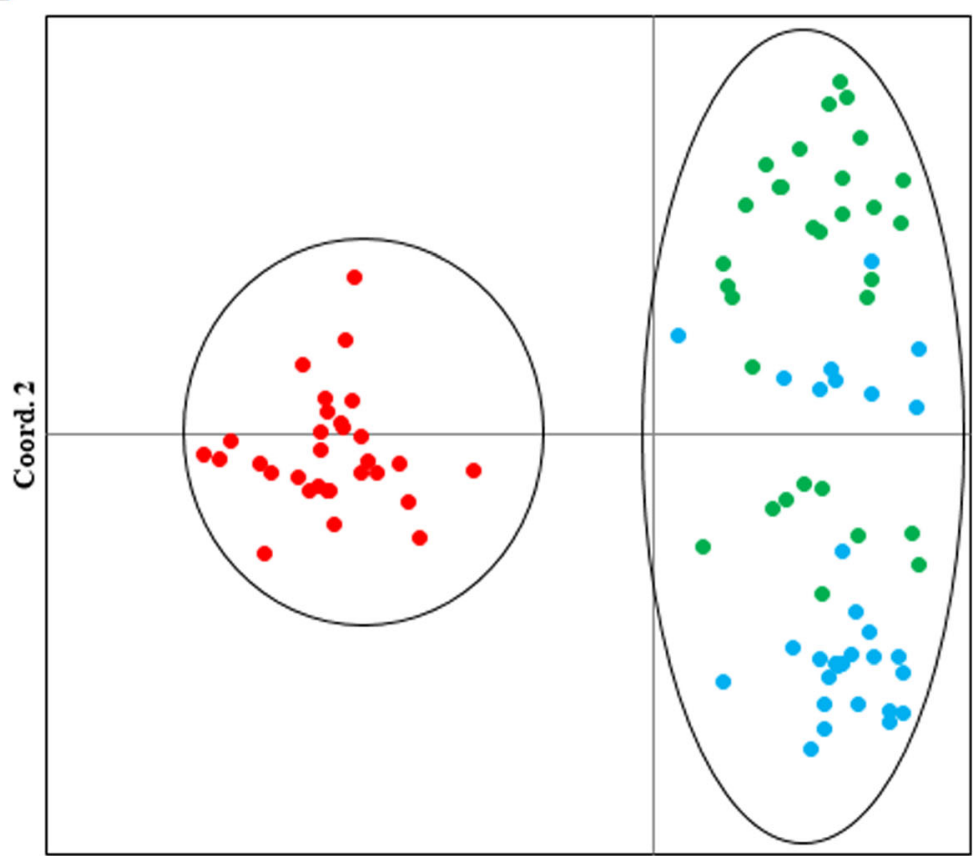

Coord. 1

- T. aestivum

- Ae crassa

- Ae. cylindrica

Fig. 4 Principal coordinate analysis (PCOA) using SCOT (panel a) and CBDP (panel b)

among the evaluated Aegilops species, whereas the lower level of diversity belonged to Ae. crassa, which was in accordance with our findings in this study. However, this result disagrees with Etminan et al. [41], so these authors reported a high level of genetic diversity in $T$. aestivum and Ae. crassa then Ae. cylindrica using CBDP markers. These contradictions could be referred to the primer's sequences, the geographical origins, or sample size of the tested accessions. Khodaee et al. [50] also reported a high level of genetic diversity among the Iranian Ae. triuncialis 
accessions using ISSR, CBDP, and SCoT molecular markers and confirmed that all the three marker systems can provide a comprehensive pattern of the genetic diversity in Ae. triuncialis germplasm.

In SCoT and CBDP analyses, clustering patterns were consistent with the results of population structure analysis. In both analyses, all investigated accessions were clustered based on their genomic structure with a minor admixture (Figs. 2, 3, and 4). Previously, a similar grouping pattern was observed for accessions from different Aegilops and Triticum species by Pour-Aboughadareh et al. [29] and Etminan et al. [41]. These authors reported SCoT and CBDP markers group species based on their genetic backgrounds and the obtained groups or subpopulations approximately confirm their taxonomic classification.

\section{Conclusion}

Preservation of the highest possible level of genetic diversity is one of the main goals of genetic resource conservation programs and assessment of genetic diversity using reliable methods provides useful information for the management of genetic resources and crop improvement programs. Our results revealed high polymorphism in the investigated Iranian wheat germplasm from different Triticum and Aegilops species. The molecular analysis of genetic diversity in the tested species showed a high level of genome variability in Ae. cylindrica species. Based on the results of AMOVA, genetic diversity observed within species was more than that found among them suggesting all accessions in each species have a wide genetic differentiation. In addition, based on obtained results, SCoT and CBDP markers were very effective techniques for the evaluation of the genetic diversity and phylogenetic studies in wheat germplasm. These results revealed that these two different genetargeted molecular markers can be used as reliable techniques for detecting the levels of DNA polymorphism and genetic relationship.

\section{Abbreviations \\ T: Triticum; Ae: Aegilops; CBDP: CAAT box-derived polymorphism; NBP: Number of polymorphic bands; I: Shannon index; ISSR: Inter-simple sequence repeats; MI: Marker index; PCoA: Principal coordinate analysis; PIC: Polymorphism information content; Rp: Resolving power; SCOT: Start codon targeted; UPGMA: Unweighted pair group method with arithmetic mean algorithm; AMOVA: Analysis of molecular variance; Na: Number of observed alleles; Ne: Number of effective alleles; He: Nei's gene diversity; AFLP: Amplified fragment length polymorphism; RAPD: Randomly amplified polymorphic DNA; SSR: Simple Sequence Repeat; DArT: Diversity arrays technology; QTL: Quantitative trait loci}

\section{Acknowledgements}

The authors would like to acknowledge Dr. Ali Ashraf Mehrabi from llam University, Iran, and Dr. Alireza Pour-aboughadareh from Seed and Plant Improvement Institute, Iran, for providing some plant materials.

\section{Authors' contributions}

$A E$ and $L S$ conceived the research idea and designed the experiments. GG, $A E, A M$, and $L S$ performed the experiments and analyzed the data. GG and
LS wrote the manuscript. AE revised and approved the final manuscript. The author(s) read and approved the final manuscript.

Funding

Not applicable.

Availability of data and materials

All data generated or analyzed during this study are included in this published article.

\section{Declarations}

Ethics approval and consent to participate

This article does not contain any studies with human participants or animals performed.

\section{Consent for publication}

Not applicable.

\section{Competing interests}

The authors declare that they have no competing interests.

Received: 28 January 2021 Accepted: 30 March 2021

Published online: 14 April 2021

\section{References}

1. International Grains Council (2019) Five-year baseline projections of supply and demand for wheat, maize (corn), rice and soyabeans to 2023/24

2. Nowak JL, Okon S, Wieremczuk A (2020) Molecular diversity analysis of genotypes from four Aegilops species based on retrotransposonmicrosatellite amplifed polymorphism (REMAP) markers. Cereal Res Commun. 49(1):37-44. https://doi.org/10.1007/s42976-020-00086-1

3. Hegde SG, Valkoun J, Waines JG (2002) Genetic diversity in wild and weedy Aegilops, Amblyopyrum, and Secale species-a preliminary survey. Crop Sci 42(2):608-614. https://doi.org/10.2135/cropsci2002.6080

4. Baum BR, Edwards T, Johnson DA (2009) Phylogenetic relationships among diploid Aegilops species inferred from 55 rDNA units. Mol Phylogenet Evol 53(1):34-44. https://doi.org/10.1016/j.ympev.2009.06.005

5. Zaharieva M, Gaulin E, Havaux M, Acevedo E, Monneveux P (2001) Drought and heat responses in the wild wheat relative Aegilops geniculate Roth: potential interest for wheat improvement. Crop Sci 41(4):1321-1329. https:// doi.org/10.2135/cropsci2001.4141321x

6. Dhanda S, Sethi GS, Behl K (2004) Indices of drought tolerance in wheat genotypes at early stages of plant growth. J Agron Crop Sci 190(1):6-12. https://doi.org/10.1111/j.1439-037X.2004.00592.x

7. Schneider A, Molnar I, Molnar-Lang M (2008) Utilisation of Aegilops (goatgrass) species to widen the genetic diversity of cultivated wheat. Euphytica 163(1):1-19. https://doi.org/10.1007/s10681-007-9624-y

8. Yesayan AH, Grigorin KV, Danielian AM, Hovhannisyan NA (2009) Determination of salt tolerance in wild einkorn wheat (Triticum boeoticum Boiss.) under in vitro conditions. Crop Wild Relatives 7:4-7

9. Hovhannisyan NA, Dulloo ME, Yesayan AH, Knupffer H, Amri A (2011) Tracking of powdery mildew and leaf rust resistance genes in Triticum boeoticum and T. urartu, wild relatives of common wheat. Czech Genet Plant 47(2):45-57. https://doi.org/10.17221/127/2010-CJGPB

10. Arabbeigi M, Arzani A, Majidi MM, Kiani R, Tabatabaei BES, Habibi F (2014) Salinity tolerance ofAegilops cylindrica genotypes collected from hypersaline shores of Uremia Salt Lake using physiological traits and SSR markers. Acta Physiol Plant 36(8):2243-2251. https://doi.org/10.1007/s11738-014-1 602-0

11. Pour-Aboughadareh A, Ahmadi J, Mehrabi AA, Etminan A, Moghaddam M, Siddique KHM (2017a) Physiological responses to drought stress in wild relatives of wheat: implications for wheat improvement. Acta Physiol Plant 39(4):106. https://doi.org/10.1007/s11738-017-2403-z

12. Pour-Aboughadareh A, Ahmadi J, Mehrabi AA, Moghaddam M, Etminan A (2017c) Evaluation of agro-morphological diversity in wild relatives of wheat collected in Iran. J Agric Sci Technol 19:943-956

13. Pour-Aboughadareh A, Omidi M, Naghavi MR, Etminan A, Mehrabi AA, Poczai P, Bayat H (2019) Effect of water deficit stress on seedling biomass and physio-chemical characteristics in different species of wheat possessing 
the D genome. Agronomy 9(9):522. https://doi.org/10.3390/a gronomy9090522

14. Pour-Aboughadareh A, Omidi M, Naghavi MR, Etminan A, Mehrabi AA, Poczai P (2020) Wild relative of wheat respond well to water deficit stress: a comparative study of antioxidant enzyme activities and their encoding gene expression. Agriculture 10(9):425. https://doi.org/10.3390/agriculture1 0090415

15. Ahmadi J, Pour-Aboughadareh A, Fabriki Ourang S, Mehrabi AA, Siddique KHM (2018a) Screening wheat germplasm for seedling root architectural traits under contrasting water regimes: Potential sources of variability for drought adaptation. Arch Agron Soil Sci 64(10):1351-1365. https://doi.org/1 $0.1080 / 03650340.2018 .1432855$

16. Ahmadi J, Pour-Aboughadareh A, Fabriki Ourang S, Mehrabi AA, Siddique KHM (2018b) Wild relatives of wheat: Aegilops-Triticum accessions disclose different antioxidative and physiological response to water stress. Acta Physiol Plant 40(5):90. https://doi.org/10.1007/s11738-018-2673-0

17. Ahmadi J, Pour-Aboughadareh A, Fabriki-Ourang S, Mehrabi AA, Siddique KHM (2018c) Screening wild progenitors of wheat for salinity stress at early stages of plant growth: insight into potential sources of variability for salinity adaptation in wheat. Crop Pasture Sci 69(7):649-658. https://doi. org/10.1071/CP17418

18. Ahmadi J, Pour-Aboughadareh A, Fabriki Ourang S, Khalili P, Poczai P (2020) Unraveling salinity stress responses in ancestral and neglected wheat species at early growth stage: A baseline for utilization in future wheat improvement programs. Physiol Mol Biol Plants 26(3):537-549. https://doi. org/10.1007/s12298-020-00768-4

19. Olivera PD, Rouse $M N$, Jin $Y$ (2019) Identification of new sources of resistance to wheat stem rust in Aegilops spp. in the tertiary gene pool of wheat. Front Plant Sci 9:1719. https://doi.org/10.3389/fpls.2018.01719

20. Mohammadi SA, Prasanna BM (2003) Analysis of genetic diversity in crop plants - salient statistical tools and considerations. Crop Sci 43(4):1235-1248. https://doi.org/10.2135/cropsci2003.1235

21. Pour-Aboughadareh A, Mohmoudi M, Ahmadi J, Moghaddam M, Mehrabi AA, Alavikia SS (2017d) Agro-morphological and molecular variability in Triticum boeoticum accessions from Zagros Mountains, Iran. Genet Resour Crop Evol 64(3):545-556. https://doi.org/10.1007/s10722-016-0381-4

22. Collard BCY, Mackill DJ (2009) Start codon targeted (SCOT) polymorphism: A simple, novel DNA marker technique for generating gene-targeted markers in plants. Plant Mol Biol Rep 27(1):86-93. https://doi.org/10.1007/s11105008-0060-5

23. Singh AK, Rana MK, Singh S, Kumar S, Kumar R, Singh R (2014) CAAT boxderived polymorphism (CBDP): a novel promoter-targeted molecular marker for plants. J Plant Biochem Biot 23(2):175-183. https://doi.org/10.1007/s13 562-013-0199-5

24. Satya P, Karan M, Jana S, Mitra S, Sharma A, Karmakar PG, Ray DP (2015) Start codon targeted (SCOT) polymorphism reveals genetic diversity in wild and domesticated populations of ramie (Boehmeria nivea L. Gaudich.), a premium textile fiber producing species. Meta Gene 3:62-70. https://doi. org/10.1016/.mgene.2015.01.003

25. Heikrujam M, Kumar J, Agrawal V (2015) Genetic diversity analysis among male and female Jojoba genotypes employing gene targeted molecular markers, start codon targeted (SCOT) polymorphism and CAAT boxderived polymorphism (CBDP) markers. Meta Gene 5:90-97. https://doi.org/10.1016/ j.mgene.2015.06.001

26. Etminan A, Pour-Aboughadareh A, Mohammadi R, Ahmadi-Rad A, Noori A, Mahdavian Z, Moradi Z (2016) Applicability of start codon targeted (SCOT) and inter-simple sequence repeat (ISSR) markers for genetic diversity analysis in durum wheat genotypes. Biotechnol Biotechnol Equip 30(6): 1075-1081. https://doi.org/10.1080/13102818.2016.1228478

27. Etminan A, Pour-Aboughadareh A, Noori A, Ahmadi-Rad A, Shooshtari L (2018b) Genetic relationships and diversity among wild Salvia accessions revealed by ISSR and SCOT markers. Biotechnol Biotechnol Equip 32(3):610617. https://doi.org/10.1080/13102818.2018.1447397

28. Tiwari G, Singh R, Singh N, Choudhury DR, Paliwal R, Kumar A, Gupta V (2016) Study of arbitrarily amplified (RAPD and ISSR) and gene targeted (SCOT and CBDP) markers for genetic diversity and population structure in Kalmegh [Andrographis paniculata (Burm.f.) Nees]. Ind Crops Prod 86:1-11. https://doi.org/10.1016/j.indcrop.2016.03.031

29. Pour-Aboughadareh A, Ahmadi J, Mehrabi AA, Etminan A, Moghaddam M (2018) Insight into the genetic variability analysis and relationships among some Aegilops and Triticum species, as genome progenitors of bread wheat, using SCoT markers. Plant Biosyst 152(4):694-703. https://doi.org/10.1 080/11263504.2017.1320311

30. Gogoi B, Wann SB, Saikia SP (2020) Comparative assessment of ISSR, RAPD, and SCOT markers for genetic diversity in Clerodendrum species of North East India. Mol Biol Rep 47(10):7365-7377. https://doi.org/10.1007/s11033-02 0-05792-x

31. Agarwal A, Gupta V, Haq SU, Jatav P-K, Kothari SL, Kachhwaha S (2019) Assessment of genetic diversity in 29 rose germplasms using SCOT marker. J King Saud Univ Sci 31(4):780-788. https://doi.org/10.1016/j.jksus.2018.04.022

32. Qaderi A, Omidi M, Pour-Aboughadareh A, Poczai P, Shaghani J, Mehrafarin A, Nohooji M, Etminan A (2019) Molecular diversity and phytochemical variability in the Iranian poppy (Papaver bracteatum Lindl.): A baseline for conservation and utilization in future breeding programmes. Ind Crops Prod 130:237-247. https://doi.org/10.1016/j.indcrop.2018.12.079

33. Doyle JJ, Doyle JL (1987) A rapid DNA isolation procedure for small quantities of fresh leaf tissue. Phytochemical Bulletin 19:11-15

34. Peakall R, Smouse PE (2006) GENALEX 6: genetic analysis in excel. Population genetic software for teaching and research. Mol Ecol Notes 6(1): 288-295. https://doi.org/10.1111/j.1471-8286.2005.01155.x

35. Perrier $X$, Jacquemoud-Collet J (2006) DARwin software at available at: http://darwin.cirad.fr/

36. Pritchard JK, Stephens M, Donnelly P (2000) Inference of population structure using multilocus genotype data. Genetics 155(2):945-959

37. Earl DA, vonHoldt BM (2012) Structure Harvester: a website and program for visualizing STRUCTURE output and implementing the Evanno method. Conserv Genet Resour 4(2):359-361. https://doi.org/10.1007/s12686-01 1-9548-7

38. Monneveux P, Zaharieva M, Rekika D, Royo C, Nachit MM, Fonzo ND, Araus $J L$ (2000) The utilisation of Triticum and Aegilops species for the improvement of durum wheat. Opt Mediterr. Serie A Seminaires Mediterr 40:71-81

39. Jaisankar I, Subramani T, Velmurugan A, Singh AK (2017) Genetic Diversity Revealed Among Rattan Genotypes from Andaman and Nicobar Islands Based on RAPD and ISSR Markers. Int J For Eng 1(2):00007. https://doi.org/1 0.15406/freij.2017.01.00007

40. Pour-Aboughadareh A, Ahmadi J, Mehrabi AA, Etminan A, Moghaddam M (2017b) Assessment of genetic diversity among Iranian Triticum germplasm using agro-morphological traits and start codon targeted (SCOT) markers. Cereal Res Commun 45(4):574-586. https://doi.org/10.1556/0806.45.2017.033

41. Etminan A, Pour-Aboughadareh A, Mehrabi AA, Shooshtari L, Ahmadi-Rad A, Moradkhani H (2019) Molecular characterization of the wild relatives of wheat using CAAT-box derived polymorphism. Plant Biosyst 153(3):398-405. https://doi.org/10.1080/11263504.2018.1492993

42. Powell W, Morgante M, Andre C, Hanafey M, Vogel J, Tingey S, Rafalski A (1996) The comparison of RFLP, RAPD, AFLP and SSR (microsatellite) markers for germplasm analysis. Mol Breed 2(3):225-238. https://doi.org/10.1007/ BF00564200

43. Etminan A, Pour-Aboughadareh A, Mohammadi R, Noori A, Ahmadi-Rad A (2018a) Applicability of CAAT box-derived polymorphism (CBDP) markers for analysis of genetic diversity in durum wheat. Cereal Res Commun 46(1):1-9. https://doi.org/10.1556/0806.45.2017.054

44. Naghavi MR, Ranjbar M, Zali A, Aghaei MJ, Mardi M, Pirseyedi SM (2009) Genetic diversity of Aegilops crassa and its relationship with Aegilops tauschii and the D genome of wheat. Cereal Res Commun 37(2):159-167. https://doi.org/10.1556/CRC.37.2009.2.2

45. Thomas KG, Bebeli PJ (2010) Genetic diversity of Greek Aegilops species using different types of nuclear genome markers. Mol Phylogenet Evol 56(3):951-961. https://doi.org/10.1016/j.ympev.2010.04.041

46. Wang Y, Wang C, Zhang H, Yue Z, Liu X, Ji W (2013) Genetic analysis of wheat (Triticum aestivum L.) and related species with SSR markers. Genet Resour Crop Evol 60(3):1105-1117. https://doi.org/10.1007/s10722-012-9907-6

47. Moradkhani H, Mehrabi AA, Etminan A, Pour-Aboughadareh A (2015) Molecular diversity and phylogeny of Triticum-Aegilops species possessing D genome revealed by SSR and ISSR markers. Plant Breed Seed Sci 71(1):8295. https://doi.org/10.1515/plass-2015-0024

48. Abbasov M, Brueggeman R, Raupp J, Akparov Z, Aminov N, Bedoshvili D, Gross T, Gross P, Babayeva S, Izzatullayeva V, Mammadova SA, Hajiyev E, Rustamov K, Bikram S-G (2019) Genetic diversity of Aegilops L. species from Azerbaijan and Georgia using SSR markers. Genet Resour Crop Evol 66(2): 453-463. https://doi.org/10.1007/s10722-018-0725-3

49. Econopouly B, Mckay J, Westra P, Reid S, Helm A, Byrne P (2013) Phenotypic diversity of Aegilops cylindrica (jointed goatgrass) accessions from the 
western United States under irrigated and dryland conditions. Agric Ecosyst Environ 164:244-251. https://doi.org/10.1016/j.agee.2012.10.005

50. Khodaee L, Azizinezhad R, Etminan A, Khosroshahi M (2021) Assessment of genetic diversity among Iranian Aegilops triuncialis accessions using ISSR,

SCOT, and CBDP markers. J Genet Eng Biotechnol 19(1):5. https://doi.org/1 0.1186/s43141-020-00107-w

\section{Publisher's Note}

Springer Nature remains neutral with regard to jurisdictional claims in published maps and institutional affiliations.

Submit your manuscript to a SpringerOpen ${ }^{\circ}$ journal and benefit from:

- Convenient online submission

- Rigorous peer review

- Open access: articles freely available online

High visibility within the field

- Retaining the copyright to your article

Submit your next manuscript at $\boldsymbol{\nabla}$ springeropen.com 